\title{
Optimal Transmission Policies for Variance Based Event Triggered Estimation With an Energy Harvesting Sensor
}

\author{
Alex S. Leong, Subhrakanti Dey, and Daniel E. Quevedo
}

\begin{abstract}
This paper considers a remote state estimation problem where a sensor observes a dynamical process, and transmits local state estimates over an independent and identically distributed (i.i.d.) packet dropping channel to a remote estimator. The sensor is equipped with energy harvesting capabilities. At every discrete time instant, provided there is enough battery energy, the sensor decides whether it should transmit or not, in order to minimize the expected estimation error covariance at the remote estimator. For transmission schedules dependent only on the estimation error covariance at the remote estimator, the energy available at the sensor, and the harvested energy, we establish structural results on the optimal scheduling which show that for a given battery energy level and a given harvested energy, the optimal policy is a threshold policy on the error covariance, i.e. transmit if and only if the error covariance exceeds a certain threshold. Similarly, for a given error covariance and a given harvested energy, the optimal policy is a threshold policy on the battery level. Numerical studies confirm the qualitative behaviour predicted by our structural results.
\end{abstract}

\section{INTRODUCTION}

In event triggered estimation, a sensor will transmit measurements to a remote estimator only when certain events occur, e.g. if the estimation quality has deteriorated sufficiently [1]-[4]. By transmitting only when necessary in order to achieve certain performance objectives, energy savings can be achieved, which are important for sensors operating in low energy environments such as wireless sensor networks. Different strategies such as transmitting when the estimation error [1], functions of the estimation error [3], or the estimation error covariance [2], [4] exceeds a threshold, have been considered. For variance based triggering (where transmit decisions depend on the estimation error covariance), it was shown in [4] that a threshold policy is optimal, in the sense that it minimizes a convex combination of the expected estimation error covariance and expected energy usage.

The harvesting of energy from the surrounding environment such as solar, thermal, or electromagnetic radiation, has been proposed as a solution to the limited battery life of sensors, which may need to operate continuously for many years in sensor network applications. Transmission strategies for optimizing communication objectives such as maximizing throughput or minimizing transmission delay have been extensively studied, see e.g. [5]-[8]. More recently, transmission

A. Leong and D. Quevedo are with the Department of Electrical Engineering (EIM-E), Paderborn University, Paderborn, Germany. E-mail: alex.leong@upb.de, dquevedodieee.org. S. Dey is with the Department of Engineering Science, Uppsala University, Uppsala, Sweden. E-mail: Subhra.Dey@signal.uu.se. strategies for optimizing estimation and control objectives such as minimizing expected estimation error covariance and LQG costs have also received attention, see [9]-[11].

The focus of this paper is to study an event triggered remote estimation problem where the sensor is equipped with energy harvesting capabilities, and transmission over a packet dropping channel can only occur if there is sufficient energy in the battery. In particular, we wish to derive structural results on the optimal transmission policy that minimizes the expected estimation error covariance at the remote estimator. For noiseless measurements and no packet drops, some structural results have been previously derived in [12]. In this paper, we will prove that for a given battery energy level and a given harvested energy, the optimal policy is a threshold policy on the error covariance (with the threshold dependent on the battery level and energy harvested). Similarly, for a given error covariance and a given harvested energy, the optimal policy is a threshold policy on the battery level.

The paper is organized as follows. Section II gives the system model. The optimal transmission scheduling problem is formulated in Section III. Structural results for the optimal transmission schedule are derived in Section IV. Some numerical results verifying the behaviour predicted by our structural results are presented in Section $\mathrm{V}$.

\section{SYSTEM MODEL}

A diagram of the system model is shown in Fig. 1. Consider a discrete time process

$$
x_{k+1}=A x_{k}+w_{k}
$$

where $x_{k} \in \mathbb{R}^{n}$ and $w_{k}$ is i.i.d. Gaussian with zero mean and covariance $Q$. The sensor has measurements

$$
y_{k}=C x_{k}+v_{k}
$$

where $y_{k} \in \mathbb{R}^{n}$ and $v_{k}$ is Gaussian with zero mean and covariance $R$. The noise processes $\left\{w_{k}\right\}$ and $\left\{v_{k}\right\}$ are assumed to be mutually independent.

\section{A. Smart Sensor with Energy Harvesting}

The sensor has computational capabilities (i.e. the sensor is "smart") and can run a local Kalman filter. The local state estimates and error covariances

$$
\begin{aligned}
\hat{x}_{k \mid k-1}^{s} & \triangleq \mathbb{E}\left[x_{k} \mid y_{0}, \ldots, y_{k-1}\right], \quad \hat{x}_{k \mid k}^{s} \triangleq \mathbb{E}\left[x_{k} \mid y_{0}, \ldots, y_{k}\right] \\
P_{k \mid k-1}^{s} & \triangleq \mathbb{E}\left[\left(x_{k}-\hat{x}_{k \mid k-1}^{s}\right)\left(x_{k}-\hat{x}_{k \mid k-1}^{s}\right)^{T} \mid y_{0}, \ldots, y_{k-1}\right] \\
P_{k \mid k}^{s} & \triangleq \mathbb{E}\left[\left(x_{k}-\hat{x}_{k \mid k}^{s}\right)\left(x_{k}-\hat{x}_{k \mid k}^{s}\right)^{T} \mid y_{0}, \ldots, y_{k}\right]
\end{aligned}
$$




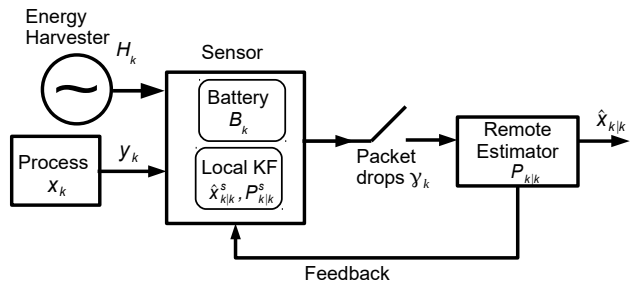

Fig. 1. Remote State Estimation with an Energy Harvesting Sensor

can be computed at the sensor using the standard Kalman filtering equations, see e.g. [13]. We will assume that the pair $(A, C)$ is detectable and the pair $\left(A, Q^{1 / 2}\right)$ is stabilizable. Let $\bar{P}$ be the steady state value of $P_{k \mid k}^{s}$ as $k \rightarrow \infty$, which exists due to the detectability assumptions [13]. To simplify the presentation, we will assume that the local Kalman filter is operating in the steady state regime, so that $P_{k \mid k}^{s}=\bar{P}, \forall k$.

Let $\nu_{k} \in\{0,1\}$ be decision variables such that $\nu_{k}=1$ if and only if $\hat{x}_{k \mid k}^{s}$ is to be transmitted to the remote estimator at time $k$. Let $B_{k}$ denote the battery level of the sensor at time $k$, with $B_{\max }$ the maximum capacity of the battery. There is an energy usage of $E$ for each scheduled transmission. Transmission can only occur if there is sufficient battery energy, i.e. $\nu_{k}=1$ is possible only when $B_{k} \geq E$. The sensor is equipped with energy harvesting capabilities, with the energy harvested between the discrete time instants $k-1$ and $k$ denoted by $H_{k}$. Similar to [7], the evolution of the battery level is modelled as

$B_{k+1}=\min \left\{B_{k}-\nu_{k} E+H_{k+1}, B_{\max }\right\}=g\left(B_{k}-\nu_{k} E+H_{k+1}\right)$

with $\nu_{k}=0$ if $B_{k}<E$, where the function $g($.$) is defined by$

$$
g(x) \triangleq \min \left\{x, B_{\max }\right\}
$$

The energy harvested process $\left\{H_{k}\right\}$ will be assumed to be a Markov process, with state space $\mathbb{H}$. Also denote $\mathbb{B} \triangleq$ $\left[0, B_{\max }\right]$. The decision variables $\nu_{k}$ are determined at the sensor, and in this paper will be assumed to depend on $P_{k-1 \mid k-1}, H_{k}$ and $B_{k}$.

At time instances when $\nu_{k}=1$, the sensor transmits its local state estimate $\hat{x}_{k \mid k}^{s}$ over a packet dropping channel. Let $\gamma_{k}$ be random variables such that $\gamma_{k}=1$ if the transmission at time $k$ is successfully received by the remote estimator, and $\gamma_{k}=0$ otherwise. We will assume that $\left\{\gamma_{k}\right\}$ is i.i.d. Bernoulli [14] with

$$
\mathbb{P}\left(\gamma_{k}=1\right)=\lambda \in(0,1) .
$$

\section{B. Optimal Remote Estimator}

At instances where $\nu_{k}=1$, it is assumed that the remote estimator knows whether the transmission was successful or not, i.e., the remote estimator knows the value $\gamma_{k}$, with dropped packets discarded. On the other hand, if $\nu_{k}=0$, since the sensor is not scheduled to transmit at this time, the corresponding $\gamma_{k}$ is assumed to be of no use to the remote estimator. Define

$$
\mathcal{I}_{k} \triangleq\left\{\nu_{0}, \ldots, \nu_{k}, \nu_{0} \gamma_{0}, \ldots, \nu_{k} \gamma_{k}, \nu_{0} \gamma_{0} \hat{x}_{0 \mid 0}^{s}, \ldots, \nu_{k} \gamma_{k} \hat{x}_{k \mid k}^{s}\right\}
$$

as the information set available to the remote estimator at time $k$. Denote the state estimates and error covariances at the remote estimator by:

$$
\hat{x}_{k \mid k} \triangleq \mathbb{E}\left[x_{k} \mid \mathcal{I}_{k}\right], P_{k \mid k} \triangleq \mathbb{E}\left[\left(x_{k}-\hat{x}_{k \mid k}\right)\left(x_{k}-\hat{x}_{k \mid k}\right)^{T} \mid \mathcal{I}_{k}\right]
$$

Given that the decision variables $\nu_{k}$ depend on $P_{k-1 \mid k-1}, H_{k}$ and $B_{k}$, but not on the state $x_{k}$, the optimal remote estimator can be shown to have the following form [15], [16]:

$$
\begin{aligned}
& \hat{x}_{k \mid k}=\left\{\begin{array}{cc}
A \hat{x}_{k-1 \mid k-1}, & \nu_{k} \gamma_{k}=0 \\
\hat{x}_{k \mid k}^{s}, & \nu_{k} \gamma_{k}=1
\end{array}\right. \\
& P_{k \mid k}=\left\{\begin{array}{cc}
f\left(P_{k-1 \mid k-1}\right) & , \quad \nu_{k} \gamma_{k}=0 \\
\bar{P} & , \quad \nu_{k} \gamma_{k}=1
\end{array}\right.
\end{aligned}
$$

where

$$
f(X) \triangleq A X A^{T}+Q .
$$

We assume that $\gamma_{k}$ is fed back to the sensor before the transmission decision at the next time instant $k+1$. Thus, the remote estimate $P_{k \mid k}$ can be reconstructed at the sensor with this acknowledgement mechanism. ${ }^{1}$

\section{OPTIMIZATION OF TRANSMISSION SCHEDULING}

In this section we will formulate a suitable optimization problem for determining the transmission schedule that minimizes the expected error covariance subject to energy harvesting constraints. Structural properties of the optimal solution to this problem will then be derived in Section IV.

Define the countable set

$$
\mathcal{S} \triangleq\left\{\bar{P}, f(\bar{P}), f^{2}(\bar{P}), \ldots\right\},
$$

where $f^{n}($.$) is the n$-fold composition of $f($.$) , with the$ convention that $f^{0}(X)=X$. Then it is clear from (6) that $\mathcal{S}$ consists of all possible values of $P_{k \mid k}$ at the remote estimator. As mentioned in Section II, we will consider transmission policies where $\nu_{k}$ depends only on $P_{k-1 \mid k-1}, H_{k}$ and $B_{k}$. We will consider the following optimization problem of finite horizon $K$ :

$$
\begin{aligned}
& \min _{\substack{\nu_{k} \in\{0,1\} \\
\nu_{k} E \leq B_{k}}} \sum_{k=1}^{K} \mathbb{E}\left[\operatorname{tr} P_{k \mid k}\right] \\
& =\min _{\substack{\nu_{k} \in\{0,1\} \\
\nu_{k} E \leq B_{k}}} \sum_{k=1}^{K} \mathbb{E}\left[\mathbb{E}\left[\operatorname{tr} P_{k \mid k} \mid P_{k-1 \mid k-1}, \nu_{k}, H_{k}, B_{k}\right]\right]
\end{aligned}
$$

We note that

$$
\begin{aligned}
& \mathbb{E}\left[\operatorname{tr} P_{k \mid k} \mid P_{k-1 \mid k-1}, \nu_{k}, H_{k}, B_{k}\right] \\
& =\nu_{k}\left[\lambda \operatorname{tr}(\bar{P})+(1-\lambda) \operatorname{tr} f\left(P_{k-1 \mid k-1}\right)\right]+\left(1-\nu_{k}\right) \operatorname{tr} f\left(P_{k-1 \mid k-1}\right) \\
& =\nu_{k} \lambda \operatorname{tr}(\bar{P})+\left(1-\nu_{k} \lambda\right) \operatorname{tr} f\left(P_{k-1 \mid k-1}\right)
\end{aligned}
$$

\footnotetext{
${ }^{1}$ The case of imperfect feedback acknowledgements can also be considered, using similar ideas as in [9].
} 
Let the functions $J_{k}(\cdot, \cdot, \cdot): \mathcal{S} \times \mathbb{H} \times \mathbb{B} \rightarrow \mathbb{R}$ be defined recursively as:

$$
\begin{aligned}
& J_{K+1}(P, H, B)=0 \\
& J_{k}(P, H, B)=\min _{\substack{\nu \in\{0,1\} \\
\nu E \leq B}}\{\nu \lambda \operatorname{tr}(\bar{P})+(1-\nu \lambda) \operatorname{tr} f(P) \\
& \quad+\nu \lambda \mathbb{E}\left[J_{k+1}(\bar{P}, \tilde{H}, g(B-\nu E+\tilde{H})) \mid H\right] \\
& \left.\quad+(1-\nu \lambda) \mathbb{E}\left[J_{k+1}(f(P), \tilde{H}, g(B-\nu E+\tilde{H})) \mid H\right]\right\}
\end{aligned}
$$

for $k=K, \ldots, 1$, where the conditional expectations are with respect to $\tilde{H}$ given $H$, and $g($.$) is defined in (4). Problem (9)$ can be solved using the dynamic programming algorithm, by computing $J_{k}\left(P_{k-1 \mid k-1}, H_{k}, B_{k}\right)$ for $k=K, K-1, \ldots, 1$. Note that if the range of $H_{k}$ is continuous, then in practice $H_{k}$ and $B_{k}$ will need to be discretized in order for problem (9) to be solved numerically.

\section{Structural PROPERTIES OF Optimal TRANSMISSION SCHEDULING}

Numerical solutions to the optimization problem (9) via dynamic programming do not provide much insight into the form of the optimal solution. In this section, we will derive some structural results on the optimal solutions to problem (9). To be more specific, we show that for a given $B_{k}$ and $H_{k}$, the optimal policy is a threshold policy with respect to the error covariance $P_{k-1 \mid k-1}$, i.e. it is optimal to transmit if and only if $P_{k-1 \mid k-1}$ exceeds a certain threshold (dependent on $k, B_{k}$ and $H_{k}$ ). Similarly, for fixed $P_{k-1 \mid k-1}$ and $H_{k}$, the optimal policy is a threshold policy with respect to the battery level $B_{k}$. Knowing that the optimal policies are of threshold-type can also provide computational savings when solving problem (9) numerically, see e.g. the discussion in [17].

\section{A. Preliminaries}

For symmetric matrices $X$ and $Y$, we say that $X \leq Y$ if $Y-X$ is positive semi-definite, and $X<Y$ if $Y-X$ is positive definite. In general, " $\leq$ " only gives a partial ordering on the set of positive semi-definite matrices. However, when restricted to $\mathcal{S}$ defined in (8), we have the following result.

Lemma IV.1. There is a total ordering on the elements of $\mathcal{S}$ given by

$$
\bar{P} \leq f(\bar{P}) \leq f^{2}(\bar{P}) \leq \ldots
$$

A proof of Lemma IV.1 may be found in [4]. We will say that a function $F():. \mathcal{S} \rightarrow \mathbb{R}$ is increasing if

$$
X \leq Y \Rightarrow F(X) \leq F(Y) .
$$

Lemma IV.2. For any $n \in \mathbb{N}, \operatorname{tr} f^{n}(P)$ is an increasing function of $P$.

Proof. We have

$$
\operatorname{tr} f^{n}(P)=\operatorname{tr}\left(A^{n} P\left(A^{n}\right)^{T}+\sum_{m=0}^{n-1} A^{m} Q\left(A^{m}\right)^{T}\right)
$$

which is increasing with $P$.

Lemma IV.3. For $d \geq 0$, the function $g($.$) defined in (4)$ satisfies

$$
0 \leq g(x)-g(x-d) \leq d
$$

Proof. The inequality $g(x)-g(x-d) \geq 0$ is obvious. For the other inequality, note that if $x \leq B_{\max }$, then $g(x)-g(x-$ $d)=x-(x-d)=d$. If $x>B_{\max }$ and $x-d>B_{\max }$, then $g(x)-g(x-d)=B_{\max }-B_{\max }=0$. If $x>B_{\max }$ (which implies $x-d>B_{\max }-d$ ) and $x-d \leq B_{\max }$, then $g(x)-g(x-d)=B_{\max }-(x-d)<B_{\max }-\left(B_{\max }-d\right)=d$.

\section{B. Threshold Policies with Respect to Error Covariance}

We have the following result on the optimality of threshold policies with respect to the remote estimator error covariance.

Theorem IV.4. For fixed $B_{k}$ and $H_{k}$, the optimal $\nu_{k}^{*}$ is a threshold policy on $P_{k-1 \mid k-1}$ of the form:

$$
\nu_{k}^{*}\left(P_{k-1 \mid k-1}, B_{k}, H_{k}\right)= \begin{cases}0 \quad, \quad P_{k-1 \mid k-1} \leq P^{*} \\ 1 \quad, \quad \text { otherwise }\end{cases}
$$

where the threshold $P^{*}$ depends on $k, P_{k-1 \mid k-1}$ and $H_{k}$.

Proof. For $B \geq E, J_{k}(P, H, B)$ in (10) can be expressed as

$$
\begin{aligned}
& J_{k}(P, H, B)=\min \left\{\operatorname{tr} f(P)+\mathbb{E}\left[J_{k+1}(f(P), \tilde{H}, g(B+\tilde{H})) \mid H\right],\right. \\
& \lambda \operatorname{tr} \bar{P}+(1-\lambda) \operatorname{tr} f(P)+\lambda \mathbb{E}\left[J_{k+1}(\bar{P}, \tilde{H}, g(B-E+\tilde{H})) \mid H\right] \\
& \left.+(1-\lambda) \mathbb{E}\left[J_{k+1}(f(P), \tilde{H}, g(B-E+\tilde{H})) \mid H\right]\right\},
\end{aligned}
$$

corresponding to the values $\nu_{k}=0$ or $\nu_{k}=1$. Since $\nu_{k}$ only takes on the two values 0 and 1, Theorem IV.4 will be proved if we can show that for fixed $B \geq E$ and $H$, the functions

$$
\begin{aligned}
\phi_{k} & (P) \triangleq \operatorname{tr} f(P)+\mathbb{E}\left[J_{k+1}(f(P), \tilde{H}, g(B+\tilde{H})) \mid H\right]-\lambda \operatorname{tr} \bar{P} \\
& -(1-\lambda) \operatorname{tr} f(P)-\lambda \mathbb{E}\left[J_{k+1}(\bar{P}, \tilde{H}, g(B-E+\tilde{H})) \mid H\right] \\
& -(1-\lambda) \mathbb{E}\left[J_{k+1}(f(P), \tilde{H}, g(B-E+\tilde{H})) \mid H\right], \\
= & \lambda\left(\operatorname{tr} f(P)-\operatorname{tr} \bar{P}-\mathbb{E}\left[J_{k+1}(\bar{P}, \tilde{H}, g(B-E+\tilde{H})) \mid H\right]\right) \\
& +\mathbb{E}\left[J_{k+1}(f(P), \tilde{H}, g(B+\tilde{H})) \mid H\right] \\
& -(1-\lambda) \mathbb{E}\left[J_{k+1}(f(P), \tilde{H}, g(B-E+\tilde{H})) \mid H\right]
\end{aligned}
$$

for $k=1, \ldots, K$ are increasing functions of $P$. Since $\operatorname{tr} f(P)$ is increasing with $P$ by Lemma IV.2, this will be the case if we can show that $\mathbb{E}\left[J_{k}(f(P), \tilde{H}, g(B+\tilde{H})) \mid H\right]-(1-$ $\lambda) \mathbb{E}\left[J_{k}(f(P), \tilde{H}, g(B-E+\tilde{H})) \mid H\right]$ is an increasing function of $P$ for all $k$.

In fact, we will prove the stronger statement that

$$
J_{k}\left(f^{n}(P), H, B\right)-(1-\lambda) J_{k}\left(f^{n}(P), H, B^{\prime}\right)
$$

is an increasing function of $P$ for all $k \in\{1, \ldots, K+1\}$, $n \in \mathbb{N}, H \geq 0, B \geq 0, B^{\prime} \geq 0$ with $0 \leq B-B^{\prime} \leq E$, noting that $0 \leq g(B+\tilde{H})-g(B-E+\tilde{H}) \leq E$ by Lemma IV.3. In order to show that (12) is an increasing function of $P$, it turns out that we also need to show that

$$
J_{k}\left(f^{n}(P), H, B^{\prime}\right)-J_{k}\left(f^{n}(P), H, B\right)
$$


is an increasing function of $P$ for all $k \in\{1, \ldots, K+1\}$, $n \in \mathbb{N}, H \geq 0, B \geq 0, B^{\prime} \geq 0$ with $0 \leq B-B^{\prime} \leq E$.

The proof is by induction. That (12) and (13) are increasing functions of $P$ in the case of $k=K+1$ is clear. For $P \geq P^{\prime}$ and $0 \leq B-B^{\prime} \leq E$, assume that

$$
\begin{aligned}
& J_{l}\left(f^{n}(P), H, B\right)-(1-\lambda) J_{l}\left(f^{n}(P), H, B^{\prime}\right) \\
& -J_{l}\left(f^{n}\left(P^{\prime}\right), H, B\right)+(1-\lambda) J_{l}\left(f^{n}\left(P^{\prime}\right), H, B^{\prime}\right) \geq 0
\end{aligned}
$$

and

$$
\begin{aligned}
& J_{l}\left(f^{n}(P), H, B^{\prime}\right)-J_{l}\left(f^{n}(P), H, B\right) \\
& -J_{l}\left(f^{n}\left(P^{\prime}\right), H, B^{\prime}\right)+J_{l}\left(f^{n}\left(P^{\prime}\right), H, B\right) \geq 0
\end{aligned}
$$

holds for $l=K+1, K, \ldots, k+1$.

Let us first show that (14) holds for $l=k$. We have

$$
\begin{aligned}
& J_{k}(\left.f^{n}(P), H, B\right)-(1-\lambda) J_{k}\left(f^{n}(P), H, B^{\prime}\right) \\
&- J_{k}\left(f^{n}\left(P^{\prime}\right), H, B\right)+(1-\lambda) J_{k}\left(f^{n}\left(P^{\prime}\right), H, B^{\prime}\right) \\
&=\min _{\nu, \nu E \leq B}\left\{\nu \lambda \operatorname{tr}(\bar{P})+(1-\nu \lambda) \operatorname{tr} f^{n+1}(P)\right. \\
& \quad+\nu \lambda \mathbb{E}\left[J_{k+1}(\bar{P}, \tilde{H}, g(B-\nu E+\tilde{H})) \mid H\right] \\
&\left.\quad+(1-\nu \lambda) \mathbb{E}\left[J_{k+1}\left(f^{n+1}(P), \tilde{H}, g(B-\nu E+\tilde{H})\right) \mid H\right]\right\} \\
&-(1-\lambda) \min _{\nu, \nu E \leq B^{\prime}}\left\{\nu \lambda \operatorname{tr}(\bar{P})+(1-\nu \lambda) \operatorname{tr} f^{n+1}(P)\right. \\
& \quad+\nu \lambda \mathbb{E}\left[J_{k+1}\left(\bar{P}, \tilde{H}, g\left(B^{\prime}-\nu E+\tilde{H}\right)\right) \mid H\right] \\
&\left.\quad+(1-\nu \lambda) \mathbb{E}\left[J_{k+1}\left(f^{n+1}(P), \tilde{H}, g\left(B^{\prime}-\nu E+\tilde{H}\right)\right) \mid H\right]\right\} \\
&-\min _{\nu, \nu E}\left\{\nu \lambda \operatorname{tr}(\bar{P})+(1-\nu \lambda) \operatorname{tr} f^{n+1}\left(P^{\prime}\right)\right. \\
& \quad+\nu \lambda \mathbb{E}\left[J_{k+1}(\bar{P}, \tilde{H}, g(B-\nu E+\tilde{H})) \mid H\right] \\
&\left.\quad+(1-\nu \lambda) \mathbb{E}\left[J_{k+1}\left(f^{n+1}\left(P^{\prime}\right), \tilde{H}, g(B-\nu E+\tilde{H})\right) \mid H\right]\right\} \\
&+(1-\lambda) \min _{\nu, \nu E \leq B^{\prime}}\left\{\nu \lambda \operatorname{tr}(\bar{P})+(1-\nu \lambda) \operatorname{tr} f^{n+1}\left(P^{\prime}\right)\right. \\
& \quad+\nu \lambda \mathbb{E}\left[J_{k+1}\left(\bar{P}, \tilde{H}, g\left(B^{\prime}-\nu E+\tilde{H}\right)\right) \mid H\right] \\
&\left.\quad+(1-\nu \lambda) \mathbb{E}\left[J_{k+1}\left(f^{n+1}\left(P^{\prime}\right), \tilde{H}, g\left(B^{\prime}-\nu E+\tilde{H}\right)\right) \mid H\right]\right\}
\end{aligned}
$$

If $B \geq E$ and $B^{\prime} \geq E$, then

$$
\begin{aligned}
J_{k} & \left(f^{n}(P), H, B\right)-(1-\lambda) J_{k}\left(f^{n}(P), H, B^{\prime}\right) \\
- & J_{k}\left(f^{n}\left(P^{\prime}\right), H, B\right)+(1-\lambda) J_{k}\left(f^{n}\left(P^{\prime}\right), H, B^{\prime}\right) \\
\geq & \min _{\nu}\left\{(1-\nu \lambda) \operatorname{tr} f^{n+1}(P)\right. \\
& +(1-\nu \lambda) \mathbb{E}\left[J_{k+1}\left(f^{n+1}(P), \tilde{H}, g(B-\nu E+\tilde{H})\right) \mid H\right] \\
& -(1-\lambda)\left[(1-\nu \lambda) \operatorname{tr} f^{n+1}(P)\right. \\
& \left.-(1-\nu \lambda) \mathbb{E}\left[J_{k+1}\left(f^{n+1}(P), \tilde{H}, g\left(B^{\prime}-\nu E+\tilde{H}\right)\right) \mid H\right]\right] \\
& -(1-\nu \lambda) \operatorname{tr} f^{n+1}\left(P^{\prime}\right) \\
& -(1-\nu \lambda) \mathbb{E}\left[J_{k+1}\left(f^{n+1}\left(P^{\prime}\right), \tilde{H}, g(B-\nu E+\tilde{H})\right) \mid H\right] \\
& +(1-\lambda)\left[(1-\nu \lambda) \operatorname{tr} f^{n+1}\left(P^{\prime}\right)\right. \\
& \left.\left.+(1-\nu \lambda) \mathbb{E}\left[J_{k+1}\left(f^{n+1}\left(P^{\prime}\right), \tilde{H}, g\left(B^{\prime}-\nu E+\tilde{H}\right)\right) \mid H\right]\right]\right\}
\end{aligned}
$$

$$
\begin{aligned}
= & \min _{\nu}(1-\nu \lambda)\left\{\lambda\left[\operatorname{tr} f^{n+1}(P)-\operatorname{tr} f^{n+1}\left(P^{\prime}\right)\right]\right. \\
& +\mathbb{E}\left[J_{k+1}\left(f^{n+1}(P), \tilde{H}, g(B-\nu E+\tilde{H})\right) \mid H\right] \\
& -(1-\lambda) \mathbb{E}\left[J_{k+1}\left(f^{n+1}(P), \tilde{H}, g\left(B^{\prime}-\nu E+\tilde{H}\right)\right) \mid H\right] \\
& -\mathbb{E}\left[J_{k+1}\left(f^{n+1}\left(P^{\prime}\right), \tilde{H}, g(B-\nu E+\tilde{H})\right) \mid H\right] \\
& \left.+(1-\lambda) \mathbb{E}\left[J_{k+1}\left(f^{n+1}\left(P^{\prime}\right), \tilde{H}, g\left(B^{\prime}-\nu E+\tilde{H}\right)\right) \mid H\right]\right\} \\
\geq & 0,
\end{aligned}
$$

where the last inequality holds (for both cases $\nu=0$ and $\nu=1$ ) by Lemma IV.2 and the induction hypothesis (14), since $0 \leq g(B-\nu E+\tilde{H})-g\left(B^{\prime}-\nu E+\tilde{H}\right) \leq E$ when $0 \leq B-B^{\prime} \leq E$.

If $B<E$ and $B^{\prime}<E$, or if $B \geq E$ and $B^{\prime}<E$, then the inequality $J_{k}\left(f^{n}(P), H, B\right)-(1-\lambda) J_{k}\left(f^{n}(P), H, B^{\prime}\right)-$ $J_{k}\left(f^{n}\left(P^{\prime}\right), H, B\right)+(1-\lambda) J_{k}\left(f^{n}\left(P^{\prime}\right), H, B^{\prime}\right) \geq 0$ can be shown by similar arguments, with the case $B \geq E$ and $B^{\prime}<E$ also making use of the induction hypothesis (15). This proves that (14) holds for $l=k$.

It remains to show that (15) holds for $l=k$. This can be done using the same method as showing that (14) holds for $l=k$, and will make use of both induction hypotheses (15) and (14). The details are omitted for brevity.

\section{Threshold Policies with Respect to Battery Level}

We also have the following result on the optimality of threshold policies with respect to the battery level:

Theorem IV.5. For fixed $P_{k-1 \mid k-1}$ and $H_{k}$, the optimal $\nu_{k}^{*}$ is a threshold policy on $B_{k}$ of the form:

$$
\nu_{k}^{*}\left(P_{k-1 \mid k-1}, B_{k}, H_{k}\right)= \begin{cases}0 & , \quad B_{k} \leq B^{*} \\ 1, & \text { otherwise }\end{cases}
$$

where the threshold $B^{*}$ depends on $k, P_{k-1 \mid k-1}$ and $H_{k}$.

Theorem IV.5 can be regarded as a special case of Theorem 6.1 of [9]. Due to paper length constraints, the details are omitted.

\section{Numerical StUdies}

We consider an example with parameters

$$
A=\left[\begin{array}{ll}
1.1 & 0.2 \\
0.2 & 0.8
\end{array}\right], C=\left[\begin{array}{ll}
1 & 1
\end{array}\right], Q=I, R=1,
$$

in which case $\bar{P}$ is easily computed as

$$
\bar{P}=\left[\begin{array}{rr}
1.3762 & -0.9014 \\
-0.9014 & 1.1867
\end{array}\right] \text {. }
$$

The packet reception probability is chosen to be $\lambda=0.7$. The transmission energy $E=2$. The energy harvested process $\left\{H_{k}\right\}$ is chosen to be i.i.d. with $H_{k}$ uniformly distributed between 0 and 2 . The maximum battery capacity $B_{\max }=6$. We use the finite horizon $K=10$.

Since $\left\{H_{k}\right\}$ is i.i.d. the optimal transmission policy will be independent of $H_{k}$. Fig. 2 plots $\nu_{k}^{*}$ for different values of $P_{k-1 \mid k-1}=f^{n}(\bar{P})$, for fixed $k=2$ and $B_{k}=2$. We observe a 


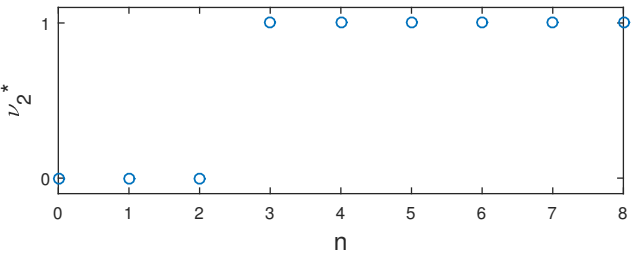

Fig. 2. $\nu_{2}^{*}$ for different values of $P_{k-1 \mid k-1}=f^{n}(\bar{P})$, for fixed $B_{k}=2$.

threshold behaviour in $P_{k-1 \mid k-1}$. Note that the plot is discrete as $P_{k-1 \mid k-1}$ lies in the discrete set $\mathcal{S}$. Fig. 3 plots $\nu_{k}^{*}$ for different values of $B_{k}$, for fixed $k=3$ and $P_{k-1 \mid k-1}=f(\bar{P})$. We now observe a threshold behaviour in $B_{k}$.

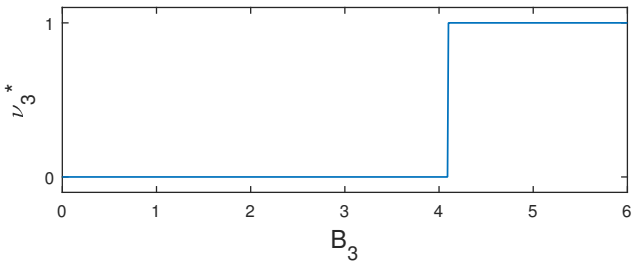

Fig. 3. $\nu_{3}^{*}$ for different values of $B_{3}$, for fixed $P_{k-1 \mid k-1}=f(\bar{P})$.

Next, we consider the case where the maximum battery capacity $B_{\max }$ is varied. Fig. 4 plots the trace of the expected error covariance $\operatorname{tr} \mathbb{E}\left[P_{k \mid k}\right]$ vs. $B_{\max }$, with $\operatorname{tr} \mathbb{E}\left[P_{k \mid k}\right]$ obtained by averaging over 100000 Monte Carlo runs, with each run having the initial values $P_{0 \mid 0}=\bar{P}$ and $B_{1}=E$. We compare the performance with a suboptimal scheme that always transmits provided there is enough energy available, i.e. $\nu_{k}=1$ if $B_{k} \geq E$. We see that the optimal solution significantly outperforms this suboptimal scheme.

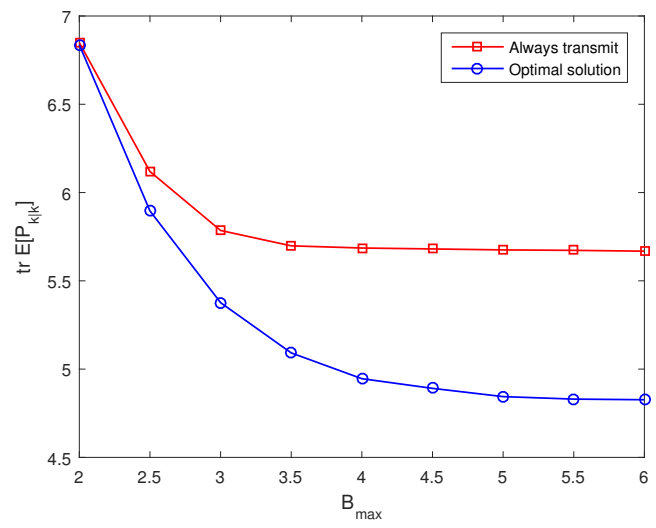

Fig. 4. Expected error covariance vs. maximum battery capacity.

\section{CONCLUSION}

This paper has considered an event triggered remote estimation problem, where the sensor is equipped with energy harvesting capabilities and transmission is over a stochastic packet dropping link. We have derived structural results on the optimal transmission scheduling in order to minimize an expected error covariance measure. Our results show that for the class of problems studied threshold policies in the error covariance and battery level are optimal.

\section{REFERENCES}

[1] L. Li, M. Lemmon, and X. Wang, "Event-triggered state estimation in vector linear processes," in Proc. American Contr. Conf., Baltimore, MD, Jun. 2010, pp. 2138-2143.

[2] S. Trimpe and R. D'Andrea, "Event-based state estimation with variance-based triggering," IEEE Trans. Autom. Control, vol. 59, no. 12, pp. 3266-3281, Dec. 2014.

[3] J. Wu, Q.-S. Jia, K. H. Johansson, and L. Shi, "Event-based sensor data scheduling: Trade-off between communication rate and estimation quality," IEEE Trans. Autom. Control, vol. 58, no. 4, pp. 1041-1046, Apr. 2013.

[4] A. S. Leong, S. Dey, and D. E. Quevedo, "On the optimality of threshold policies in event triggered estimation with packet drops," in Proc. Europ. Contr. Conf., Linz, Austria, Jul. 2015, pp. 921-927.

[5] V. Sharma, U. Mukherji, V. Joseph, and S. Gupta, "Optimal energy management policies for energy harvesting sensor nodes," IEEE Trans. Wireless Commun., vol. 9, no. 4, pp. 1326-1336, Apr. 2010.

[6] O. Ozel, K. Tutuncuoglu, J. Yang, S. Ulukus, and A. Yener, "Transmission with energy harvesting nodes in fading wireless channels: Optimal policies," IEEE J. Sel. Areas Commun., vol. 29, no. 8, pp. 1732-1743, 2011.

[7] C. K. Ho and R. Zhang, "Optimal energy allocation for wireless communications with energy harvesting constraints," IEEE Trans. Signal Process., vol. 60, no. 9, pp. 4808-4818, Sep. 2012.

[8] K. Tutuncuoglu and A. Yener, "Optimum transmission policies for battery limited energy harvesting nodes," IEEE Trans. Wireless Commun., vol. 11, no. 3, pp. 1180-1189, Mar. 2012.

[9] M. Nourian, A. S. Leong, and S. Dey, "Optimal energy allocation for Kalman filtering over packet dropping links with imperfect acknowledgments and energy harvesting constraints," IEEE Trans. Autom. Control, vol. 59, no. 8, pp. 2128-2143, Aug. 2014.

[10] Y. Li, D. E. Quevedo, V. Lau, S. Dey, and L. Shi, "Transmission power scheduling for energy harvesting sensor in remote state estimation," in Proc. IFAC World Congress, Cape Town, South Africa, Aug. 2014, pp. 122-127.

[11] S. Knorn and S. Dey, "Optimal sensor transmission energy allocation for linear control over a packet dropping link with energy harvesting," in Proc. IEEE Conf. Decision and Control, Osaka, Japan, Dec. 2015, pp. 1199-1204.

[12] A. Nayyar, T. Başar, D. Teneketzis, and V. V. Veeravalli, "Optimal strategies for communication and remote estimation with an energy harvesting sensor," IEEE Trans. Autom. Control, vol. 58, no. 9, pp. 2246-2260, Sep. 2013.

[13] B. D. O. Anderson and J. B. Moore, Optimal Filtering. New Jersey: Prentice Hall, 1979.

[14] B. Sinopoli, L. Schenato, M. Franceschetti, K. Poolla, M. I. Jordan, and S. S. Sastry, "Kalman filtering with intermittent observations," IEEE Trans. Autom. Control, vol. 49, no. 9, pp. 1453-1464, September 2004.

[15] Y. Xu and J. P. Hespanha, "Estimation under uncontrolled and controlled communications in networked control systems," in Proc. IEEE Conf. Decision and Control, Seville, Spain, December 2005, pp. 842-847.

[16] L. Schenato, "Optimal estimation in networked control systems subject to random delay and packet drop," IEEE Trans. Autom. Control, vol. 53, no. 5, pp. 1311-1317, Jun. 2008.

[17] M. H. Ngo and V. Krishnamurthy, "Optimality of threshold policies for transmission scheduling in correlated fading channels," IEEE Trans. Commun., vol. 57, no. 8, pp. 2474-2483, Aug. 2009. 